\title{
Elastic $(\mathbf{h} \boldsymbol{\mu})_{1 s}+\mathbf{H e}^{++}$Scattering and the Influence of Adiabatic Corrections on $(\mathrm{He} \mu \mathrm{h})^{++}$Bound States
}

\author{
J. Gronowski, W. Czapliński and N. Popov* \\ Faculty of Physics and Applied Computer Science \\ University of Science and Technology, AGH \\ al. Mickiewicza 30, 30-059 Kraków, Poland
}

(Received July 26, 2004; revised version November 3, 2004)

\begin{abstract}
Cross-sections for elastic scattering of muonic hydrogen on helium nuclei, $(\mathrm{h} \mu)_{1 s}+\mathrm{He}^{++}$, where $\mathrm{h}$ and $\mathrm{He}^{++}$stands for a hydrogen and a helium isotope nucleus, respectively, were calculated in the one-level adiabatic approximation for a range of collision energies from 0 to $50 \mathrm{eV}$. Bound states and energy levels of $(\mathrm{h} \mu \mathrm{He})^{++}$molecular ions were also calculated and compared with their Born-Oppenheimer counterparts. It is shown that adiabatic corrections are responsible for proper positions of the RamsauerTownsend minima in $(\mathrm{h} \mu)_{1 s}+\mathrm{He}^{++}$elastic scattering and, at the same time, they significantly influence bound states and energy levels of $(\mathrm{He} \mu \mathrm{h})^{++}$and $(\mathrm{He} \pi \mathrm{h})^{++}$ions. Calculations were performed in the frame of the phase-function method.
\end{abstract}

PACS numbers: $34.60 .+\mathrm{z}, 36.10 . \mathrm{Gv}$

\section{Introduction}

Atomic and molecular processes induced by negative muons in gaseous hydrogen and hydrogen-helium mixtures have been studied for over five decades [1]. The studies have been motivated by possible practical use of energy released in nuclear syntheses occurring inside muonic molecular ions, e.g. $(\mathrm{d} \mu \mathrm{t})^{+}$or $\left({ }^{3} \mathrm{He} \mu \mathrm{d}\right)^{++}$ (hence the common name of the processes, the muon catalyzed fusion $(\mu \mathrm{CF})[2])$

\footnotetext{
*Visiting professor at the Faculty of Physics and Applied Computer Science, University of Science and Technology, AGH.
} 
and by such fundamental questions of physics as isotope invariance and charge symmetry of strong interactions at very slow collisions ${ }^{\dagger}[3]$. At the same time, the $\mu \mathrm{CF}$ processes form an unavoidable background in experimental study of weak muon capture by hydrogen and helium isotope nuclei [4]. To extract the corresponding capture rates from different spin states of muonic atoms their spin-states population determined by collision-induced deexcitation processes has to be known. The main $\mu \mathrm{CF}$ processes that are still imperfectly understood and need further intensive theoretical and experimental investigation are listed here:

- formation of excited muonic atoms by direct muon capture into an atomic orbit [5] or by muon transfer processes between excited states of muonic atoms [6];

- elastic scattering of the ground-state or excited muonic atoms in collisions with hydrogen atoms or molecules [7-9];

- deexcitation of muonic atoms via the Auger [10] or Coulomb deexcitation processes [11];

- formation of muonic molecules in collisions of muonic hydrogen atoms with hydrogen molecules [2] or helium atoms [12];

- nuclear synthesis reactions occurring inside muonic molecules [13]. Exemplary scheme of $\mu \mathrm{CF}$ processes in $\mathrm{D}^{3}{ }^{3} \mathrm{He}$ mixture is presented in Fig. 1.

Arrows indicating transitions are labeled by corresponding reaction rates, $\lambda=\sigma N_{0} V$, where $\sigma$ denotes cross-section, $N_{0}=4.25 \times 10^{22} \mathrm{~cm}^{-3}$ is the liquid hydrogen density (LHD), and $V$ is the collision velocity. Details about the processes can be found in Refs. [2-4, 12-14]. As indicated in Fig. 1, the muon freed after the fusion may start another $\mu \mathrm{CF}$ cycle. The number of cycles is practically limited by muon sticking to $Z>1$ nuclei initially present in a mixture or produced in synthesis reactions. Theoretical study of $\mu \mathrm{CF}$ processes comprises different theoretical methods of atomic and molecular physics, e.g. variational methods, adiabatic spherical $[15,16]$ and hyper-spherical expansion method [17], quantum Monte Carlo [18], and Faddeev equations [19]. A lot of important results for $\mathrm{h}-\mu-\mathrm{He}^{++}$systems have been obtained using adiabatic expansion method [16], in particular, for quasi-stationary states, $(\mathrm{h} \mu \mathrm{He})^{++}$, usually called muonic molecules. These states correspond to very narrow Feshbach-type resonances in $\mathrm{h}+(\mathrm{He} \mu)_{1 s}^{+}$scattering for the total angular momentum of the three particle $J=0$, 1 , and 2. Positions of the resonances were calculated in Refs. [20-22] in the frame of the nonadiabatic coupled-rearrangement-channel, the two-channel hyper-spherical expansion method, and complex-coordinate-rotation method, respectively (see also the review article [23]). The resonances are situated from $0.134 \mathrm{eV}$ to $81.335 \mathrm{eV}$ below $(\mathrm{h} \mu)_{1 s}$ threshold. Widths of the resonances for $\mathrm{h}=\mathrm{d}$ are of order of $10^{-4} \mathrm{eV}$ for both ${ }^{3} \mathrm{He}$ and ${ }^{4} \mathrm{He}[20]$. The corresponding quasi-stationary states, $(\mathrm{h} \mu \mathrm{He})_{J}^{++}$, have a rich experimental evidence. They are formed in room temperature $\mathrm{H}-\mathrm{He}$ gaseous targets due to collision of the ground state muonic hydrogen with a helium

\footnotetext{
${ }^{\dagger}$ Collision energy of nuclei inside muonic molecules estimated from the uncertainty principle is of order of $1 \mathrm{keV}$. Such slow collisions are not reachable in accelerator experiments.
} 


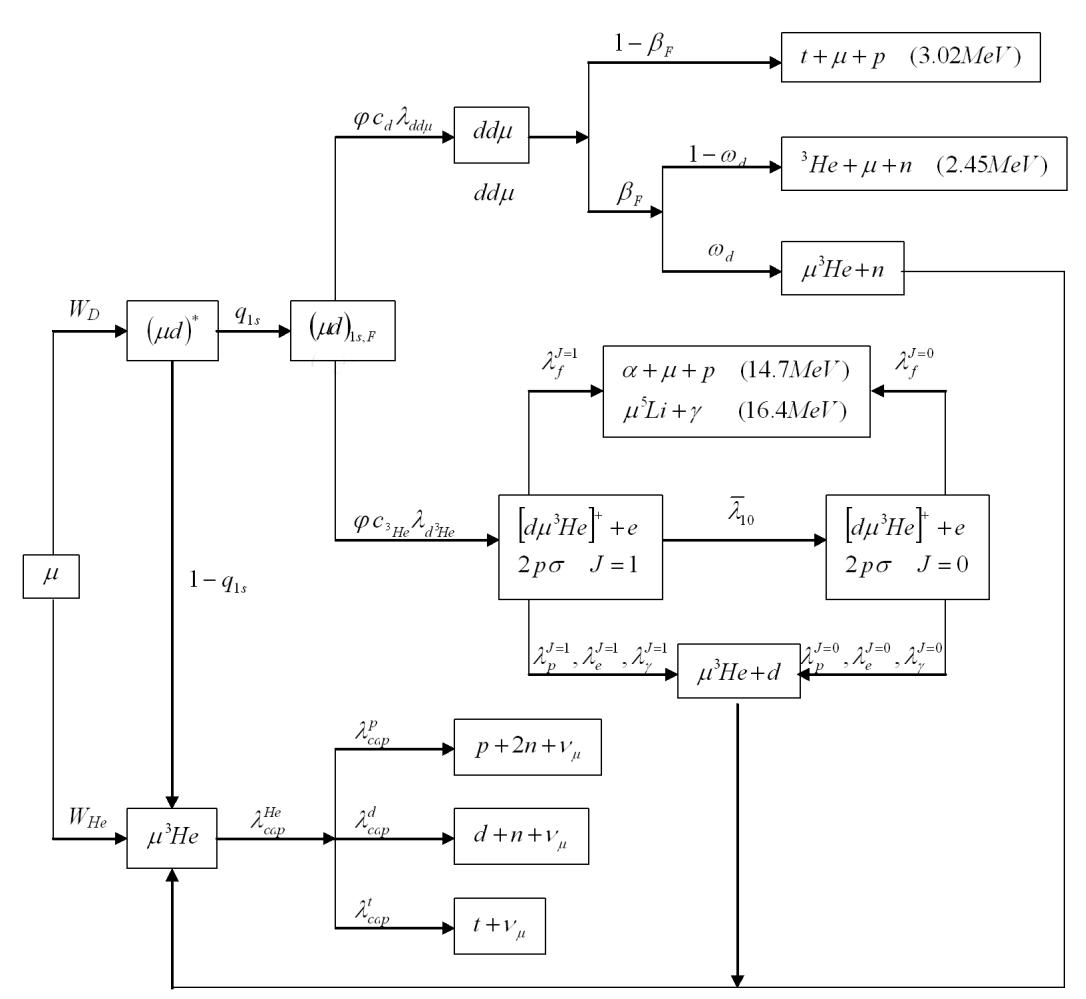

Fig. 1. Scheme of $\mu$-atomic and $\mu$-molecular processes occurring in $\mathrm{D}^{3}{ }^{3} \mathrm{He}$ mixture exposed on negative muons beam.

atom [12]:

$$
(\mathrm{h} \mu)_{1 s}+\mathrm{He} \rightarrow\left[(\mathrm{He} \mu \mathrm{h})_{J}^{++}, \mathrm{e}\right]^{+}+\mathrm{e} .
$$

The subsequent radiative decay of the resonances

$$
(\mathrm{He} \mu \mathrm{h})_{J}^{++} \rightarrow(\mathrm{He} \mu)_{1 s}^{+}+\mathrm{h}+\gamma,
$$

which result in muon transfer to helium, were observed in a lot of experiments [24] by measuring of the corresponding $\gamma$-radiation spectra.

Much broader resonances in $\mathrm{d}-\mu-{ }^{4} \mathrm{He}^{++}$and $\mathrm{t}-\mu-{ }^{4} \mathrm{He}^{++}$systems with the total angular momentum $J=0$ and 1 (of Feshbach-type also) were found in Refs. [25] and [26]. The resonances are situated from $345.6 \mathrm{eV}$ to $355.3 \mathrm{eV}$ below $(\alpha \mu)_{n=4}$ threshold [25] and from $96.0 \mathrm{eV}$ to $0.6 \mathrm{eV}$ below $(\mathrm{t} \mu)_{2 s}$ threshold [26]. They correspond to excited quasi-stationary states of $\left({ }^{4} \mathrm{He} \mu \mathrm{h}\right)_{J}^{++}$system with both decay channels, $(\mathrm{He} \mu)^{+}+\mathrm{h}$ and $(\mathrm{h} \mu)+\mathrm{He}^{++}$, open. A possible formation mechanism of such states in $\mathrm{H}-\mathrm{He}$ gaseous mixtures was proposed in Ref. [26], however, as far as we know, it has no experimental evidence still. Simple calculation shows that all of the resonances are located by more than $1.4 \mathrm{keV}$ 
above $(\mathrm{h} \mu)_{1 s}$ thresholds. Therefore, one can state a question about an existence of resonances located just above $(\mathrm{h} \mu)_{1 s}$ thresholds. We answer this question in the present paper.

The two channels involved in calculation in paper [21], $\mathrm{h}+(\mathrm{He} \mu)_{1 s}^{+}$and $(\mathrm{h} \mu)_{1 s}+\mathrm{He}^{++}$, correspond to $1 s \sigma$ and $2 p \sigma$ muon states of the two-center Coulomb problem, respectively.

However, it is possible to obtain quite accurate ro-vibrational energy levels, $\varepsilon_{J v}$, of the quasi-stationary states using the one-level adiabatic approximation for the $2 p \sigma$ state. It is due to the fact that coupling terms of the potential matrix corresponding to the two-level treatment are much smaller than the diagonal ones in this region of the inter-nuclear separation, $R \geq 4 a_{\mu}$ ( $a_{\mu}$ is the muonic atom Bohr radius), where the $2 p \sigma$-component of the total wave function is localized [27]. The corresponding results, presented for the first time in Ref. [12], differ by several percent only from the ones obtained later by several authors using variational method for many-thousand-terms trial wave functions [20, 22, 26], as well as the two-level approximation [21, 27, 28].

At the same time, the adiabatic one-level approximation seems also to be valid in calculation of elastic cross-sections for slow $(\mathrm{h} \mu)_{1 s}+\mathrm{He}^{++}$collisions. Justification of the method is the same here as for molecular states because the corresponding adiabatic $2 p \sigma$-potential has a steep slope (see Fig. 4 below). Consequently, the left-hand turning point shifts only slightly towards smaller $R$ when the energy increases from $\varepsilon_{J v}$ to about $50 \mathrm{eV}$. Cross-sections for elastic $(\mathrm{p} \mu)_{1 s}+{ }^{3} \mathrm{He}^{++}$ scattering were calculated in Ref. [29] in the frame of the two-level approximation including $s$ and $p$ partial waves. The Ramsauer-Townsend effect was observed at about $0.2 \mathrm{eV}$. On the other hand, the corresponding one-level calculation for $2 p \sigma$ state with and without inclusion of electron screening of a helium nucleus was performed in Ref. [30] in adiabatic approximation for $s$ and $p$ partial waves for all hydrogen and helium isotopes. However, the unscreened $s$-wave cross-sections for elastic $(\mathrm{p} \mu)_{1 s}+{ }^{3} \mathrm{He}^{++}$scattering significantly differ from the corresponding ones obtained in the present paper using the same method of calculation and from the two-level results of Ref. [29].

Similar, i.e. one-level calculation of elastic scattering of the ground state $\mathrm{p} \mu$ atoms from C, N, O, and F nuclei are presented in Ref. [31]. Results were obtained, however, in the Born-Oppenheimer (B-O) approximation. Due to presence of quasi-crossings of terms involved in $\mathrm{p}-\mu-Z$ systems, where $Z>2$ [32], the effective one-level potential was constructed from different molecular terms in corresponding areas of the inter-nuclear separation $R$. Low energy resonances in partial cross-sections (i.e. shape resonances) were found for collision energies below $2 \mathrm{eV}$.

This paper is arranged as follows. The adiabatic expansion method for three-body $\mathrm{h}-\mu-\mathrm{He}^{++}$Coulomb problem is shortly described in Sec. 2. The phase-function method for one-level scattering- and bound-states is described in Sec. 3. Cross-sections for $(\mathrm{h} \mu)_{1 s}+\mathrm{He}^{++}$elastic collisions calculated in the 
one-level adiabatic approximation for partial waves $J=0 \div 10$ and collision energies $0 \leq \varepsilon \leq 50 \mathrm{eV}$ are presented in Sec. 4 together with their B-O counterparts. The influence of adiabatic corrections on bound-states and energy levels of $(\mathrm{He} \mu \mathrm{h})^{++}$and $(\mathrm{He} \pi \mathrm{h})^{++}$ions are also presented in Sec. 4. The obtained results are summarized in Sec. 5 .

\section{Adiabatic expansion method for $\mathrm{h}-\mu-\mathrm{He}^{++}$system}

Coordinates describing three particle system, $\mathrm{h}-\mu-\mathrm{He}^{++}$, are presented in Fig. 2. The muon position $\boldsymbol{r}$ is reckoned from the middle of $R$. After separation of

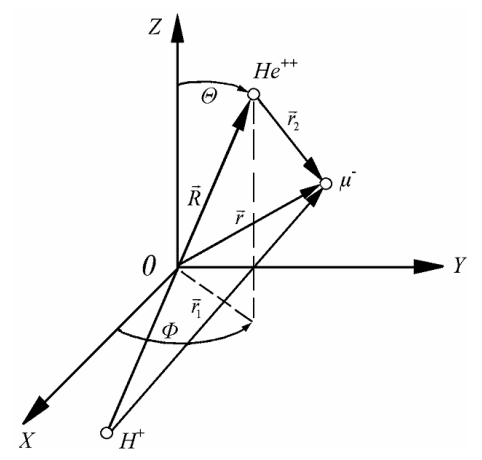

Fig. 2. Coordinates used for description of $\mathrm{He}-\mu-\mathrm{h}$ system.

the center-of-mass motion the non-relativistic Hamiltonian for $\mathrm{h}-\mu-\mathrm{He}^{++}$system receives the form (in atomic units $e=m_{\mathrm{e}}=\hbar=1$ )

$$
H=-\frac{1}{2 M}\left[\nabla_{R}+(\kappa / 2) \nabla_{r}\right]^{2}-\frac{1}{2 m_{0}} \nabla_{r}^{2}-\frac{2}{|\boldsymbol{r}+\boldsymbol{R} / 2|}-\frac{1}{|\boldsymbol{r}-\boldsymbol{R} / 2|}+\frac{2}{R},
$$

where

$$
M^{-1}=M_{\mathrm{He}}^{-1}+M_{\mathrm{h}}^{-1}, \quad m_{0}^{-1}=m_{\mu}^{-1}+\left(M_{\mathrm{He}}+M_{\mathrm{h}}\right)^{-1}
$$

and

$$
\kappa=\left(M_{\mathrm{He}}-M_{\mathrm{h}}\right) /\left(M_{\mathrm{He}}+M_{\mathrm{h}}\right) .
$$

Asymptotic form of $\mathrm{h}-\mu-\mathrm{He}^{++}$system corresponding to $(\mathrm{h} \mu)_{1 s}+\mathrm{He}^{++}$channel suggests, however, another splitting of the total Hamiltonian in which the reduced mass of the muonic atom, $m_{\mathrm{a}}^{-1}=M_{\mathrm{h}}^{-1}+m_{\mu}^{-1}$, will appear explicitly instead of $m_{0}[16]$ (below we use the $\mu$-atomic units $(\mu \mathrm{au}), e=\hbar=m_{\mathrm{a}}=1$ )

$$
H=T+H_{\mu}+\frac{2}{R}
$$

where 


$$
\begin{aligned}
& T=-\frac{1}{2 M}\left\{\left[\nabla_{R}+(\kappa / 2) \nabla_{r}\right]^{2}-[(\kappa+1) / 2]^{2} \nabla_{r}^{2}\right\}, \\
& H_{\mu}=-\frac{1}{2} \nabla_{r}^{2}-\frac{2}{|\boldsymbol{r}+\boldsymbol{R} / 2|}-\frac{1}{|\boldsymbol{r}-\boldsymbol{R} / 2|} .
\end{aligned}
$$

With total Hamiltonian (2) commutes the total angular momentum operator for the three particle system, $\boldsymbol{J}$, its $z$-axis projection, $\boldsymbol{J}_{z}$, and the total parity operator, $\boldsymbol{P}(\boldsymbol{R} \rightarrow-\boldsymbol{R}, \boldsymbol{r} \rightarrow-\boldsymbol{r})$. Eigenfunctions of the Hamiltonian in the total angular momentum representation read [16]:

$$
\Psi_{J M}^{\lambda}(\boldsymbol{r}, \boldsymbol{R})=\sum_{m=0}^{J} \mathcal{D}_{m M}^{J \lambda}(\Phi, \Theta, \varphi) F_{m}^{J \lambda}(\xi, \eta, R),
$$

where $\mathcal{D}_{m M}^{J \lambda}(\Phi, \Theta, \varphi)$, called the symmetrized Wigner functions, are eigenstates of $\boldsymbol{J}^{2}, \boldsymbol{J}_{z}$, and $\boldsymbol{P}$ operators

$$
\begin{aligned}
& \boldsymbol{J}^{2} \mathcal{D}_{m M}^{J \lambda}=J(J+1) \mathcal{D}_{m M}^{J \lambda}, \\
& \boldsymbol{J}_{z} \mathcal{D}_{m M}^{J \lambda}=M \mathcal{D}_{m M}^{J \lambda}, \\
& \boldsymbol{P} \mathcal{D}_{m M}^{J \lambda}=\lambda \mathcal{D}_{m M}^{J \lambda}, \quad \lambda= \pm(-1)^{J},
\end{aligned}
$$

and are expressed by usual Wigner functions [33], $D_{m M}^{J}(\Phi, \Theta, \varphi)$, in the following way:

$$
\begin{aligned}
& \mathcal{D}_{m M}^{J \lambda}(\Phi, \Theta, \varphi)=\sqrt{\frac{2 J+1}{16 \pi^{2}\left(1+\delta_{m 0}\right)}}\left[(-1)^{m} D_{m M}^{J}(\Phi, \Theta, 0) \exp (\mathrm{i} m \varphi)\right. \\
& \left.\quad+(-1)^{J} \lambda D_{-m M}^{J}(\Phi, \Theta, 0) \exp (-\mathrm{i} m \varphi)\right]
\end{aligned}
$$

with $m$ being an eigenvalue of $\boldsymbol{R} \cdot \boldsymbol{J} / R$ operator. The muon position, $\boldsymbol{r}$, is expressed in the body-fixed reference frame by prolate spheroidal coordinates, $\eta=\left(r_{1}-r_{2}\right) / R, \xi=\left(r_{1}+r_{2}\right) / R$, and $\varphi$ the azimuth angle measured in the plane perpendicular to $\boldsymbol{R} ;(\Theta, \Phi)$ are spherical angles of vector $\boldsymbol{R}$.

Adiabatic basis set for $\mathrm{h}-\mu-\mathrm{He}^{++}$system is formed by eigenfunctions $\phi_{i}(R ; \xi, \eta)$ of muonic Hamiltonian $H_{\mu}(R)$,

$$
H_{\mu}(R) \phi_{i}(R ; \xi, \eta)=E_{i}(R) \phi_{i}(R ; \xi, \eta)
$$

corresponding to the muon moving in the Coulomb field of the two resting nuclei, $\mathrm{h}$ and $\mathrm{He}^{++}$, separated by $R$. The index $i$ represents a set of quantum numbers describing bound- or continuous-spectrum states of the muon. Muonic bound states are usually labeled by spherical quantum numbers in the united atom classification $(R \rightarrow 0)$, i.e. $i=(N l m)$, whereas $i=\left(k_{\mu} l m\right)$ describes a continuous-spectrum state, where $k_{\mu}$ is the muon impulse at $r \rightarrow \infty$. The corresponding energy eigenval- 
ues (molecular terms) are denoted by $E_{i}(R)$. Adiabatic expansion of the "radial" function, $F_{m}^{J \lambda}(\xi, \eta, R)$, is usually written in the form [16]:

$$
\begin{gathered}
F_{m}^{J \lambda}(\xi, \eta, R)=\sum_{N=1}^{\infty} \sum_{l=0}^{N-1} \phi_{N l m}(R ; \xi, \eta) \chi_{N l m}^{J \lambda}(R) R^{-1} \\
+\sum_{l=0}^{\infty} \int_{0}^{\infty} \mathrm{d} k_{\mu} \phi_{k_{\mu} l m}(R ; \xi, \eta) \chi_{k_{\mu} l m}^{J \lambda}(R) R^{-1}
\end{gathered}
$$

where functions $\chi_{i}^{J \lambda}(R)$ describe relative motion of the nuclei.

The lower lying molecular term that supports a potential well in $\mathrm{h}-\mathrm{He}^{++}$ interaction in $\mathrm{h}-\mu-\mathrm{He}^{++}$system corresponds to $2 p \sigma$ muon state of the respective two-center Coulomb problem. At the same time, this state tends asymptotically to the ground state of $\mathrm{h} \mu$ atom. Therefore, the adiabatic approximation of $(\mathrm{He} \mu \mathrm{h})^{++}$ bound states as well as $(\mathrm{h} \mu)_{1 s}+\mathrm{He}^{++}$elastic scattering is realized here by retaining only one term in expansion (8) for $N l m=2 p \sigma$. The corresponding adiabatic wave function receives the form

$$
\Psi_{J M}(\boldsymbol{r}, \boldsymbol{R})=Y_{J M}(\Theta, \Phi) \varphi_{2 p \sigma}(R ; \xi, \eta) \chi_{2 p \sigma}^{J}(R) R^{-1},
$$

where $m=0$ and index $\lambda=+(-1)^{J}$ was omitted. By substitution of expression (9) into the Schrödinger equation with Hamiltonian (2) and after averaging over spherical angles $(\Theta, \Phi)$ and the muon $2 p \sigma$ state, one obtains the radial equation

$$
\frac{\mathrm{d}^{2}}{\mathrm{~d} R^{2}} \chi_{2 p \sigma}^{J}(R)+2 M\left[\varepsilon-V_{A}(R)-\frac{J(J+1)}{2 M R^{2}}\right] \chi_{2 p \sigma}^{J}(R)=0,
$$

where $\varepsilon=E-E_{2 p \sigma}(\infty)$ is the collision energy; $E$ is the total energy of the system; $E_{2 p \sigma}(\infty)$ is the ground state energy of h $\mu$ atom. The adiabatic potential $V_{\mathrm{A}}(R)$ reads

$$
V_{\mathrm{A}}(R)=V_{\mathrm{BO}}(R)+\frac{U_{\mathrm{A}}(R)}{2 M},
$$

where

$$
V_{\mathrm{BO}}(R)=E_{2 p \sigma}(R)-E_{2 p \sigma}(\infty)+\frac{2}{R}
$$

is the potential corresponding to the Born-Oppenheimer approximation, and

$$
U_{\mathrm{A}}(R)=\langle 2 p \sigma|2 M T| 2 p \sigma\rangle
$$

is the adiabatic correction, usually written in the form [16]:

$$
U_{\mathrm{A}}(R)=H^{+}(R)-H^{*}(R)+\kappa\left[H^{-}(R)-2 H^{*}(R)\right] .
$$

Functions $H^{ \pm}(R)$ and $H^{*}(R)$ are as follows: 


$$
\begin{aligned}
& H^{+}(R)=-\frac{3}{2 R^{2}}+\frac{1}{R^{2}}\left[4 E_{2 p \sigma}(R)+R E_{2 p \sigma}^{\prime}(R)\right]\left\langle\phi_{2 p \sigma}\left|r^{2}\right| \phi_{2 p \sigma}\right\rangle \\
& -3 R^{2}\left\langle\phi_{2 p \sigma}\left|r^{2} W\right| \phi_{2 p \sigma}\right\rangle+\left\langle\phi_{2 p \sigma}^{\prime}\left|\phi_{2 p \sigma}^{\prime}\right|\right\rangle, \\
& H^{-}(R)=-\frac{1}{R}\left[4 E_{2 p \sigma}(R)+R E_{2 p \sigma}^{\prime}(R)\right]\left\langle\phi_{2 p \sigma}|z| \phi_{2 p \sigma}\right\rangle+\frac{3}{R}\left\langle\phi_{2 p \sigma}|z W| \phi_{2 p \sigma}\right\rangle, \\
& H^{*}(R)=-\left[E_{2 p \sigma}(R)+R E_{2 p \sigma}^{\prime}(R)\right] / 2,
\end{aligned}
$$

where "prime" denotes derivative with respect to $R$,

$$
W=-2\left[\left(Z_{1}+Z_{2}\right) \xi+\left(Z_{2}-Z_{1}\right) \eta\right] / R\left(\xi^{2}-\eta^{2}\right) \quad \text { and } \quad Z_{1}=1, \quad Z_{2}=2 .
$$

Molecular term $E_{2 p \sigma}(R)$ and functions $H^{ \pm}(R), H^{*}(R)$ have the following asymptotic $(R \rightarrow \infty)$ behavior $[15,16]$ :

$$
\begin{aligned}
& E_{2 p \sigma}(R)=-\frac{1}{2}-\frac{Z_{2}}{R}-\frac{9}{4} \frac{Z_{2}^{2}}{R^{4}}+\mathrm{O}\left(R^{-6}\right), \\
& H^{+}(R)=0.25-\frac{12}{R^{4}}, \quad H^{-}(R)=0.5-\frac{27}{R^{4}}, \quad H^{*}(R)=0.25-\frac{15}{R^{4}},
\end{aligned}
$$

where the term - (9/4) $Z_{2}^{2} R^{-4}$ in Eq. (16) corresponds to the quadratic Stark-effect [34] for muonic hydrogen in the electric field of nucleus $Z_{2}$. At $R \rightarrow 0$ we have $[15,16]$ :

$$
\begin{aligned}
& E_{2 p \sigma}(0)=-\frac{\left(Z_{1}+Z_{2}\right)^{2}}{8}, \\
& H^{+}(R)=\frac{2}{R^{2}}, \quad H^{-}(0)=-\frac{3}{8}, \quad H^{*}(0)=\frac{9}{16} .
\end{aligned}
$$

Potentials $E_{2 p \sigma}(R), H^{ \pm}(R), H^{*}(R)$ and $V_{\mathrm{BO}}(R), V_{\mathrm{A}}(R)$ are presented in Fig. 3 and Fig. 4, respectively.

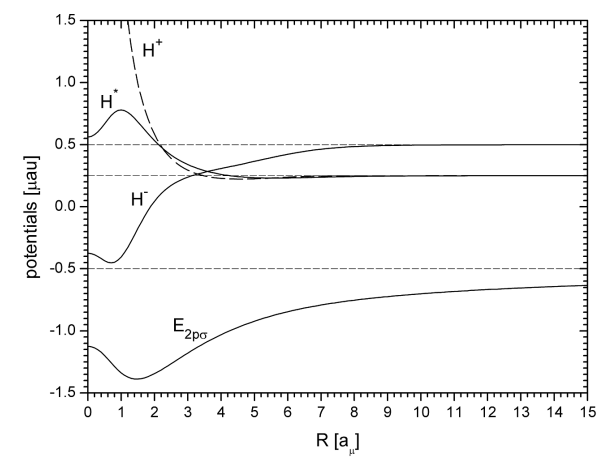

Fig. 3. Adiabatic corrections $H^{ \pm}(R), H^{*}(R)$ and molecular term $E_{2 p \sigma}(R)$ corresponding to $\mathrm{He}-\mu-\mathrm{h}$ system. 


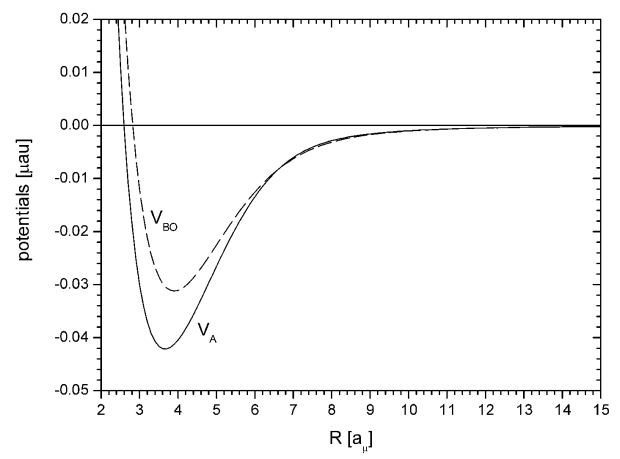

Fig. 4. The Born-Oppenheimer potential, $V_{\mathrm{BO}}$ (dashed), corresponding to any $\mathrm{h}-\mu-\mathrm{He}^{++}$system and the adiabatic potential, $V_{\mathrm{A}}$ (solid line), corresponding to $\mathrm{p}-\mu-{ }^{3} \mathrm{He}^{++}$system.

\section{Phase-function method for one-level problem}

The adiabatic wave function for $(\mathrm{h} \mu)_{1 s}+\mathrm{He}^{++}$system is the solution of Eq. (10) with the following boundary conditions (the label $2 p \sigma$ is omitted):

$$
\begin{aligned}
& \chi_{J}(0)=0, \\
& \chi_{J}(R \rightarrow \infty)=\cos \delta_{J} j_{J}(k R)-\sin \delta_{J} n_{J}(k R),
\end{aligned}
$$

where $k=\sqrt{2 M \varepsilon} ; j_{J}(k R)$ and $n_{J}(k R)$ are the Riccati-Bessel functions [35]; $\delta_{J}$ is the phase shift. Assuming the following ansatz for the radial function [36]:

$$
\chi_{J}(R)=A_{J}(R)\left[\cos \delta_{J}(k R) j_{J}(k R)-\sin \delta_{J}(k R) n_{J}(k R)\right]
$$

and the constraint

$$
\frac{\mathrm{d}}{\mathrm{d} R} \chi_{J}(R)=A_{J}(R)\left[\cos \delta_{J}(k R) \frac{\mathrm{d}}{\mathrm{d} R} j_{J}(k R)-\sin \delta_{J}(k R) \frac{\mathrm{d}}{\mathrm{d} R} n_{J}(k R)\right],
$$

one obtains the system of two coupled first-order differential equations for amplitude $A_{J}(R)$ and phase-function $\delta_{J}(R)[36]$ :

$$
\left\{\begin{array}{c}
\frac{\mathrm{d}}{\mathrm{d} r} A_{J}(R)=-\frac{2 M}{k} A_{J}(R) V(R)\left[\cos \delta_{J}(R) j_{J}(k R)-\sin \delta_{J}(R) n_{l}(k R)\right] \\
\times\left[\sin \delta_{J}(R) j_{J}(k R)+\cos \delta_{J}(R) n_{J}(k R)\right] \\
\frac{\mathrm{d}}{\mathrm{d} r} \delta_{J}(R)=-\frac{2 M}{k} V(R)\left[\cos \delta_{J}(R) j_{J}(k R)-\sin \delta_{J}(R) n_{J}(k R)\right]^{2}
\end{array}\right.
$$

with boundary conditions

$$
\begin{aligned}
& A_{J}(R \rightarrow \infty)=1, \\
& \delta_{J}(0)=0 .
\end{aligned}
$$

Equations (22) and (23) are called the amplitude and phase-equation, respectively, and together with conditions (24) and (25) are equivalent to the bound- 
ary value problem (10) and (19). Phase shift is obtained from the condition $\delta_{J}=\delta_{J}(R \rightarrow \infty)$. In order to calculate the total elastic cross-section,

$$
\sigma_{\text {tot }}=\frac{4 \pi}{k^{2}} \sum_{J=0}^{\infty}(2 J+1) \sin ^{2} \delta_{J},
$$

which is expressed in terms of phase shift only, it suffices to solve Eq. (23) with condition (25).

At the same time the fact is known that bound state energies correspond to imaginary poles of scattering $S$-matrix elements in complex $k$-plane for $\operatorname{Im}(k)>0$ [37]. The corresponding equations for the partial $S$-matrix function, $S_{J}(R)=\mathrm{e}^{\mathrm{i} \delta_{J}(R)}$, obtained from Eq. (23) and (25), receives the form [36]:

$$
\begin{aligned}
& \frac{\mathrm{d}}{\mathrm{d} r} S_{J}(R, k)=-\frac{2 M}{k} V(R)\left[h_{J}^{(-)}(k R)+\mathrm{i} S_{J}(R, k) h_{J}^{(+)}(k R)\right]^{2}, \\
& S_{J}(0, k)=1,
\end{aligned}
$$

where $h_{J}^{(+)}(k R)=j_{J}(k R) \pm \mathrm{i} n_{J}(k R)$. Bound-state energies, $\varepsilon_{n}$, can be obtained then from the requirement $S_{J}\left(\infty, \mathrm{i} \kappa_{n}\right)=\infty$, where $k=\mathrm{i} \kappa, \kappa>0, \varepsilon_{n}=-\frac{\hbar^{2} \kappa_{n}^{2}}{2 M}$, $n=0,1$, etc. For numerical purposes, however, it is convenient to replace Eq. (27) by the corresponding real equations for the real partial scattering amplitude-function defined as $f_{J}(R, k)=(-\mathrm{i})^{2 J+1} \mathrm{e}^{2 \mathrm{i} \delta_{J}(R)} \sin \delta_{J}(R)$. The equations read

$$
\begin{aligned}
& \frac{\mathrm{d}}{\mathrm{d} r} f_{J}(R, \kappa)=-\frac{2 M}{\kappa} V(R)\left[p_{J}(\kappa R)+\frac{2}{\pi} f_{J}(R, \kappa) q_{J}(\kappa R)\right]^{2}, \\
& f_{J}(0, \kappa)=0,
\end{aligned}
$$

where $p_{J}(x)=(-\mathrm{i})^{J+1} j_{J}(\mathrm{i} x)$ and $q_{J}(x)=\frac{\pi}{2 \mathrm{i}}^{J+1} h_{J}^{(+)}(\mathrm{i} x)$. The condition for bound states is $f_{J}\left(\infty, \kappa_{n}\right)=0$. Additionally, regularization of Eqs. (28) by substitution $f_{J}(R, \kappa)=\tan \left[\gamma_{J}(R, \kappa)\right]$ leads to the following boundary value problem:

$$
\begin{aligned}
& \frac{\mathrm{d}}{\mathrm{d} r} \gamma_{J}(R, \kappa)=-\frac{2 M}{\kappa} V(R)\left[p_{J}(\kappa R) \cos \gamma_{J}(R, \kappa)+\frac{2}{\pi} q_{J}(\kappa R) \sin \gamma_{J}(R, \kappa)\right]^{2} \\
& \gamma_{J}(0, \kappa)=0
\end{aligned}
$$

with the condition for bound states

$$
\gamma_{J}\left(\infty, \kappa_{n}\right)=(2 n+1) \frac{\pi}{2}
$$

Extension of the phase-function method to multi-level problems can be found in Refs. [36].

\section{Results and discussion}

\subsection{Cross-sections for elastic $(h \mu)_{1 s}+H e^{++}$scattering}

Energy dependence of several partial phase shifts for elastic $(\mathrm{p} \mu)_{1 s}+{ }^{3} \mathrm{He}^{++}$ scattering calculated in the adiabatic one-level approximation is presented in 

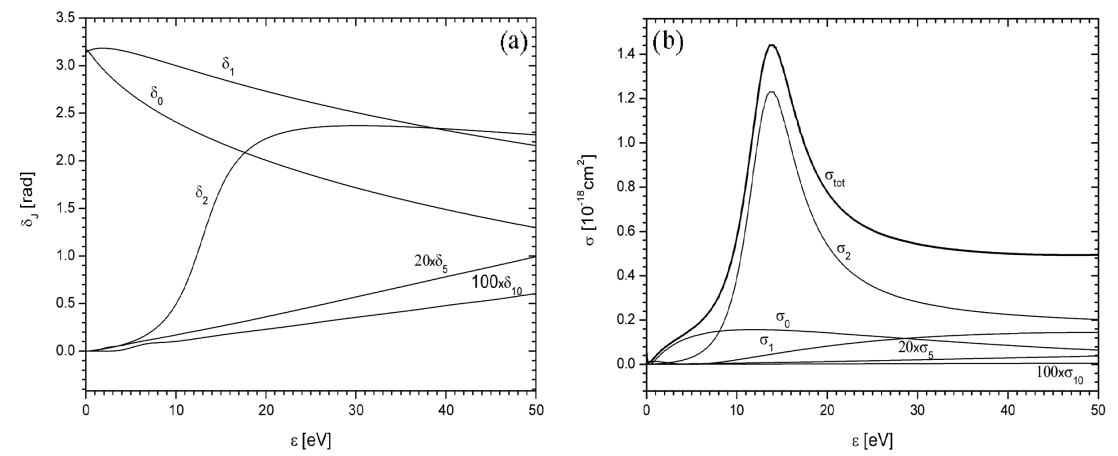

Fig. 5. (a) Partial phase shifts for elastic $(\mathrm{p} \mu)_{1 s}+{ }^{3} \mathrm{He}$ scattering vs. collision energy $\varepsilon$. (b) Partial and total cross-sections for elastic $(\mathrm{p} \mu)_{1 s}+{ }^{3}$ He scattering.

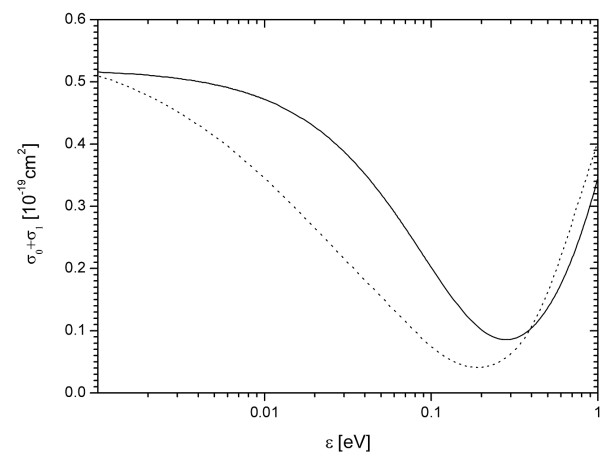

Fig. 6. Comparison of the total cross-section (including $s$ and $p$ partial waves) for elastic $(\mathrm{p} \mu)_{1 s}+{ }^{3} \mathrm{He}$ scattering calculated in the one-level adiabatic approximation in the present paper (solid line) with the corresponding total cross-section calculated in Ref. [29] in the two-level approximation (dotted line).

Fig. 5a for collision energies $0 \leq \varepsilon \leq 50 \mathrm{eV}$. Zero-energy values of phase shifts for $J=0$ and 1 , equal to $\pi$, indicate, according to the Levinson theorem $[34,37$, the existence of the corresponding one bound state of $\left({ }^{3} \mathrm{He} \mu \mathrm{p}\right)^{++}$for each $J$. The respective partial and total cross-sections are presented in Fig. 5b. According to the figure and Fig. 6, the Ramsauer-Townsend effect is observed at $\varepsilon \approx 0.28 \mathrm{eV}$, and a broad resonance corresponding to $J=3$, at $\varepsilon \approx 13 \mathrm{eV}$. The low-energy total cross-sections including dominating $s$ and $p$ partial waves are compared with the corresponding two-level results of Ref. [29] in Fig. 6. The agreement is quite satisfactory in the whole energy range considered. At the same time, our cross-sections significantly differ from the corresponding results of Ref. [30] obtained in the frame of the same method of calculation, i.e. one-level adiabatic approximation. As an illustration of the discrepancy, Fig. 7 presents the comparison of the dominating $s$-wave cross-sections obtained in the present paper with the ones of Refs. [30] and [29]. Our results are close to the corresponding two-level 


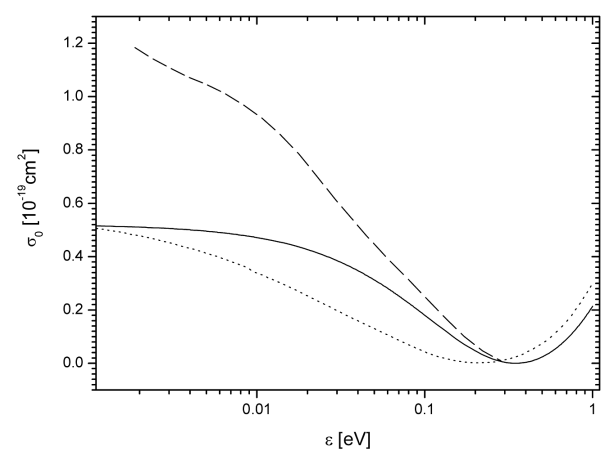

Fig. 7. Comparison of $s$-wave cross-section for elastic $(\mathrm{p} \mu)_{1 s}+{ }^{3}$ He scattering obtained in the one-level adiabatic approximation in the present paper (solid) and in Ref. [30] (dashed) with the corresponding two-level $s$-wave cross-section of Ref. [29] (dotted line).
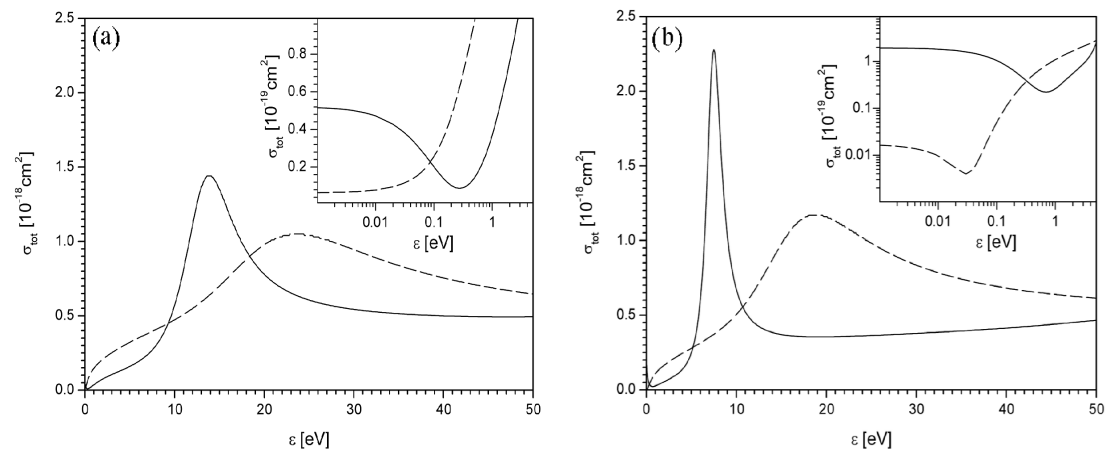

Fig. 8. (a) Comparison of the total cross-section for elastic $(\mathrm{p} \mu)_{1 s}+{ }^{3} \mathrm{He}$ scattering (partial contributions up to $J=10$ are included) obtained in the present paper with (solid) and without (dashed line) adiabatic corrections. (b) As in (a) but for elastic $(\mathrm{p} \mu)_{1 s}+{ }^{4} \mathrm{He}$ scattering.

cross-sections of Ref. [29] whereas they significantly differ from results of [30], especially for low-energy limit. We also have found a significant discrepancy between our $s$-wave elastic cross-sections and the corresponding ones of Ref. [30] for all remaining isotope compositions of $(\mathrm{h} \mu)_{1 s}+\mathrm{He}^{++}$system. The source of this discrepancy, however, is not clear to us.

Adiabatic cross-sections for $(\mathrm{p} \mu)_{1 s}+{ }^{3} \mathrm{He}^{++}$and $(\mathrm{p} \mu)_{1 s}+{ }^{4} \mathrm{He}^{++}$elastic collisions are compared with their B-O counterparts in Fig. 8a and b, respectively. As is seen from the figures, adiabatic results significantly differ from B-O ones in the whole energy range considered. At the same time, the B-O approximation significantly shifts the Ramsauer-Townsend minima towards smaller collision energies. Total elastic cross-sections for $(\mathrm{h} \mu)_{1 s}+{ }^{3} \mathrm{He}^{++}$and $(\mathrm{h} \mu)_{1 s}+{ }^{4} \mathrm{He}^{++}$, where $\mathrm{h}=\mathrm{p}, \mathrm{d}, \mathrm{t}$, calculated by summing partial contributions up to $J=10$ are collected in Figs. 9a and 10a, respectively. Very large zero-energy values for $\mathrm{h}=\mathrm{d}$ and $\mathrm{t}$ 

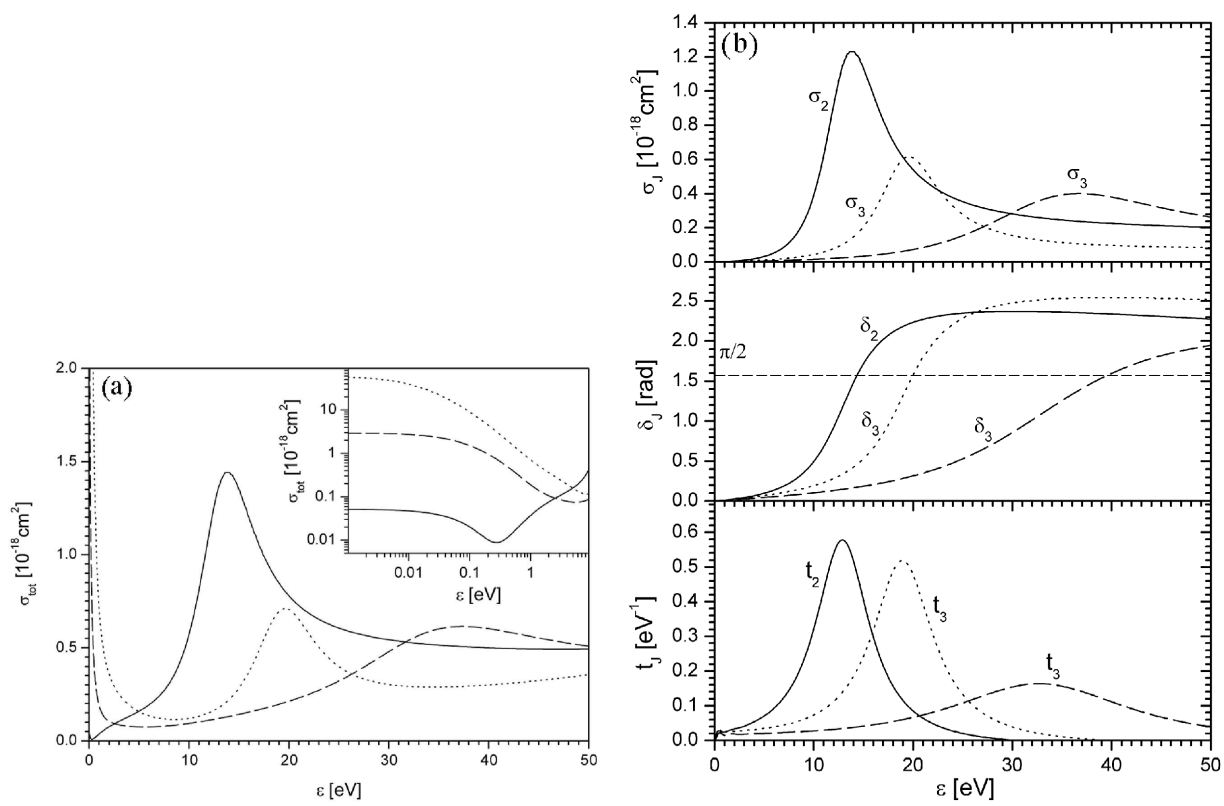

Fig. 9. (a) Energy dependence of total cross-sections for elastic $(\mathrm{h} \mu)_{1 s}+{ }^{3} \mathrm{He}$ scattering for $\mathrm{h}=\mathrm{p}$ (solid), $\mathrm{h}=\mathrm{d}$ (dashed), and $\mathrm{h}=\mathrm{t}$ (dotted line). (b) Energy dependence of partial resonance cross-sections $\left(\sigma_{J}\right)$, the respective phase shifts $\left(\delta_{J}\right)$ and time delays $\left(t_{J}\right)$, corresponding to $(\mathrm{h} \mu)_{1 s}+{ }^{3}$ He scattering. Curves are denoted as in part (a).

(only $s$-wave contribution dominates) which are visible in the insets are due to the presence of virtual (anti-bound) or weakly-bound states [38, 39]. In order to distinguish between these two possibilities we calculated the corresponding scattering lengths, $\alpha=-\lim _{k \rightarrow 0}\left(\tan \delta_{0} / k\right)$. Results are presented in Table I.

TABLE I

Scattering lengths, $\alpha$ (results in $\mu$-atomic units are presented in curly brackets), and resonance positions, $\varepsilon_{J}^{\mathrm{r}}$, and widths, $\Gamma_{J}$, obtained from Eq. (31) and from fitting (in brackets) for elastic $(\mathrm{h} \mu)_{1 s}+\mathrm{He}^{++}$scattering. Resonance partial waves for $J=2$ and $J=3$ correspond to $\mathrm{h}=\mathrm{p}$ and $\mathrm{h}=\mathrm{d}$, $\mathrm{t}$, respectively.

\begin{tabular}{c|c|c|c|c|c|c}
\hline \hline & \multicolumn{3}{|c|}{${ }^{3} \mathrm{He}$} & \multicolumn{3}{c}{${ }^{4} \mathrm{He}$} \\
\cline { 2 - 7 } & $\alpha\left[10^{-10} \mathrm{~cm}\right]$ & $\varepsilon_{J}^{\mathrm{r}}[\mathrm{eV}]$ & $\Gamma_{J}[\mathrm{eV}]$ & $\alpha\left[10^{-10} \mathrm{~cm}\right]$ & $\varepsilon_{J}^{\mathrm{r}}[\mathrm{eV}]$ & $\Gamma_{J}[\mathrm{eV}]$ \\
\hline $\mathrm{p}$ & $-0.64\{-2.3\}$ & $12.9(12.9)$ & $6.6(7.3)$ & $-1.3\{-4.4\}$ & $7.4(7.4)$ & $2.0(2.1)$ \\
$\mathrm{d}$ & $-4.9\{-18.0\}$ & $32.7(32.7)$ & $21.7(26.5)$ & $-13.0\{-48.0\}$ & $23.1(22.9)$ & $10.1(11.6)$ \\
$\mathrm{t}$ & $-21.8\{-82.1\}$ & $19.0(18.9)$ & $7.5(8.1)$ & $15.7\{59.1\}$ & $7.9(7.9)$ & $0.8(0.8)$
\end{tabular}

One sees from Table I that the low-energy elastic $(\mathrm{t} \mu)_{1 s}+{ }^{4} \mathrm{He}^{++}$cross-section is determined by the presence of a loosely bound state $(\alpha>0)$ of the 

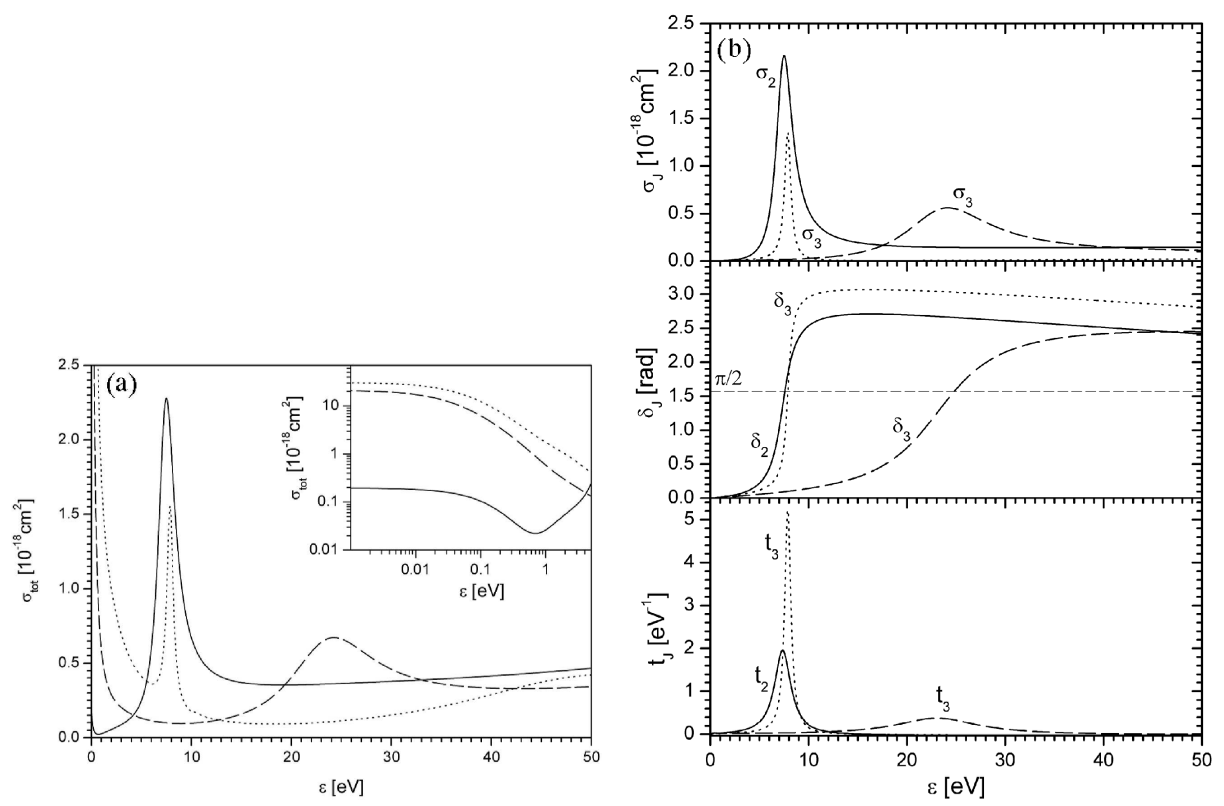

Fig. 10. (a) Energy dependence of total cross-sections for elastic $(\mathrm{h} \mu)_{1 s}+{ }^{4}$ He scattering for $\mathrm{h}=\mathrm{p}$ (solid), $\mathrm{h}=\mathrm{d}$ (dashed), and $\mathrm{h}=\mathrm{t}$ (dotted line). (b) Energy dependence of partial resonance cross-sections $\left(\sigma_{J}\right)$, the respective phase shifts $\left(\delta_{J}\right)$ and time delays $\left(t_{J}\right)$, corresponding to $(\mathrm{h} \mu)_{1 s}+{ }^{4} \mathrm{He}$ scattering. Curves are denoted as in part (a).

corresponding muonic molecule, $\left({ }^{4} \mathrm{He} \mu \mathrm{t}\right)^{++}$(see Sec. 4.2). At the same time, low-energy elastic cross-sections for the remaining hydrogen and helium isotope compositions of $(\mathrm{h} \mu)_{1 s}+\mathrm{He}^{++}$system (excluding $\mathrm{h}=\mathrm{p}$ ) are determined by the presence of virtual states $(\alpha<0)$. For $\varepsilon \gg 0$, total cross-sections exhibit distinct maxima which are due to single partial-wave resonances (shape resonances [40]) corresponding to $J=2$ for $\mathrm{h}=\mathrm{p}$ and to $J=3$ for $\mathrm{h}=\mathrm{d}$, t. The corresponding partial cross-sections, phase shifts, and time delays $\left(t_{J}=2 \mathrm{~d} \delta_{J} / \mathrm{d} E\right)$ are presented in Figs. $9 \mathrm{~b}$ and 10b. One sees from the figures that each resonance phase shift increases by about $\pi$ from a background level $\beta_{J}$. Approximate positions of the resonances, $\varepsilon_{J}^{\mathrm{r}}$, correspond to maxima of $t_{J}$. Widths of the resonances can be calculated from the equation

$$
\Gamma_{J}=\left.2 \sqrt{-2 \delta_{J}^{\prime} / \delta_{j}^{\prime \prime \prime}}\right|_{\varepsilon=\varepsilon_{J}^{\mathrm{r}}}
$$

(see Ref. [41]), where "prime" denotes the derivative with respect to $\varepsilon$. Results are also collected in Table I. As is seen from Figs. 9b, 10b, and Table I, the relation between the time delay and the resonance width, $t_{J}\left(\varepsilon_{J}^{\mathrm{r}}\right)=4 / \Gamma_{\mathrm{J}}$, is almost fulfilled for each isotope composition of $(\mathrm{h} \mu)_{1 s}+\mathrm{He}^{++}$system. At the same time, approximate resonance positions and widths calculated from fitting of the formula 


$$
\delta_{J}(\varepsilon)=\arctan \left(\frac{\varepsilon-\varepsilon_{J}^{\mathrm{r}}}{\Gamma_{J} / 2}\right)+\beta_{J}
$$

to the corresponding phase shifts are presented in Table I in parenthesis. The differences are due to energy dependence of $\beta_{J}$ neglected in Eqs. (31) and (32).

\subsection{Bound states of $(\text { He } \mu h)^{++}$molecules}

$R$-dependence of phase shift for $J=0$ corresponding to elastic $(\mathrm{t} \mu)_{1 s}+{ }^{4} \mathrm{He}^{++}$ scattering for three collision energies is presented in Fig. 11 as an example. Characteristic jumps of $\delta_{0}(R)$ by $\pi$, at $R_{1} \approx 3.8$ and $R_{2} \approx 15$, appearing when $\varepsilon \rightarrow 0$, correspond to two bound states. An abscissa of a jump equals to a minimal potential range at which the respective bound state is supported. Bound state energies were calculated by numerical solution of Eqs. (29) and (30). Function $\gamma_{0}\left(R_{\max }, \varepsilon\right)$, corresponding to elastic $(\mathrm{t} \mu)_{1 s}+{ }^{4} \mathrm{He}^{++}$scattering, obtained for $R_{\max }$, is plotted in Fig. 12. Coordinates $\varepsilon_{1}$ and $\varepsilon_{2}$, for which $\gamma_{0}\left(R_{\max }, \varepsilon\right)$ experiences successive

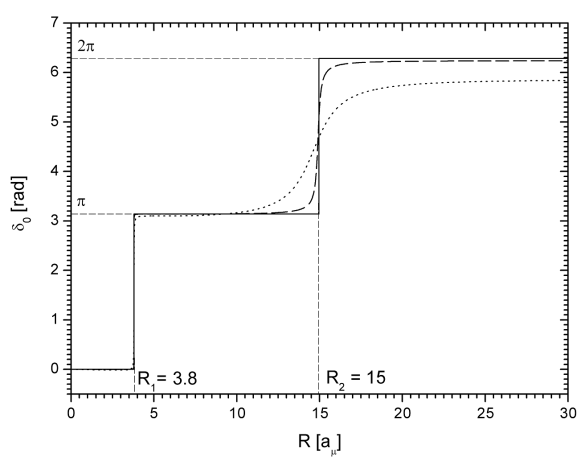

Fig. 11. $R$-dependence of phase shift, $\delta_{0}$, for elastic $(\mathrm{t} \mu)_{1 s}+{ }^{4} \mathrm{He}$ scattering for different collision energies: $\varepsilon=10^{-2} \mathrm{eV}$ (dotted), $\varepsilon=10^{-4} \mathrm{eV}$ (dashed), and $\varepsilon=10^{-10} \mathrm{eV}$ (solid line).

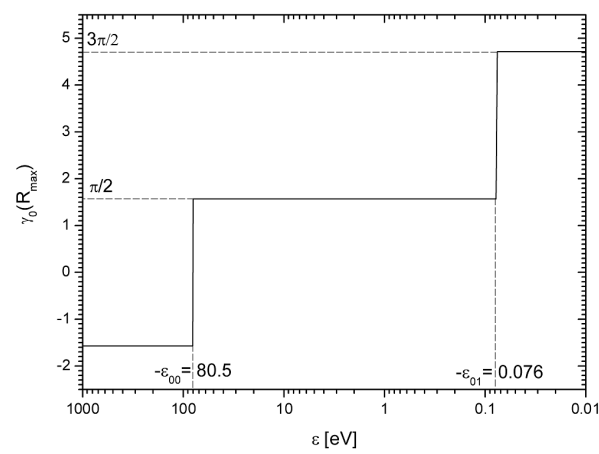

Fig. 12. Energy-dependence of function $\gamma_{0}\left(R_{\max }, \varepsilon\right)$, defined in Sec. 3, for elastic $(\mathrm{t} \mu)_{1 s}+{ }^{4}$ He scattering. 
jumps by $\pi$, are equal to binding energies of the corresponding muonic molecule for $J=0$. It is remarkable that the curves from Figs. 11 and 12 are consistent, i.e. they both indicate the presence of two bound states. Binding energies of $(\mathrm{h} \mu \mathrm{He})^{++}$molecules for all isotope combinations, with and without inclusion of adiabatic corrections are presented in Table II.

TABLE II

Energy levels of $(\mathrm{He} \mu \mathrm{h})^{++}$molecules calculated in the one-level adiabatic (Ad.) and the Born-Oppenheimer (B-O) approximation obtained in the present paper. Results obtained from two- and many-channel calculation in Refs. [27] and [26], respectively, are also presented for comparison.

\begin{tabular}{c|c|c|c|c|c|c|c}
\hline \hline$-\varepsilon_{J v}$ & & ${ }^{3} \mathrm{He} \mu \mathrm{p}$ & ${ }^{4} \mathrm{He} \mu \mathrm{p}$ & ${ }^{3} \mathrm{He} \mu \mathrm{d}$ & ${ }^{4} \mathrm{He} \mu \mathrm{d}$ & ${ }^{3} \mathrm{He} \mu \mathrm{t}$ & ${ }^{4} \mathrm{He} \mu \mathrm{t}$ \\
\hline$-\varepsilon_{00}$ & Ad. & 67.2 & 73.85 & 69.5 & 77.5 & 71.6 & 80.5 \\
& $\mathrm{~B}-\mathrm{O}$ & 39.7 & 42.3 & 59.3 & 63.5 & 68.7 & 73.8 \\
& {$[27]$} & 72.76 & 80.64 & 69.37 & 77.49 & & \\
& {$[26]$} & & & 70.976 & 79.340 & 72.296 & 81.335 \\
\hline$-\varepsilon_{01}$ & $\mathrm{Ad}$. & & & & & & 0.076 \\
& $\mathrm{~B}-\mathrm{O}$ & & & & & & 0.053 \\
& {$[26]$} & & & & & & 0.134 \\
\hline$-\varepsilon_{10}$ & $\mathrm{Ad}$. & 34.2 & 41.6 & 46.5 & 55.9 & 52.4 & 62.9 \\
& $\mathrm{~B}-\mathrm{O}$ & 13.8 & 17.1 & 37.9 & 43.6 & 49.9 & 56.9 \\
& {$[27]$} & 38.82 & 46.31 & 46.41 & 55.74 & & \\
& {$[26]$} & & & 48.419 & 58.222 & 53.330 & 63.958 \\
\hline$-\varepsilon_{20}$ & $\mathrm{Ad}$. & & & 7.25 & 17.7 & 18.2 & 30.7 \\
& $\mathrm{~B}-\mathrm{O}$ & & & 2.4 & 9.4 & 16.8 & 26.2 \\
& {$[27]$} & & & 7.11 & 17.49 & & \\
& {$[26]$} & & & 9.434 & 20.416 & 19.379 & 32.063
\end{tabular}

Our adiabatic results presented in Table II coincide almost exactly ${ }^{\ddagger}$ with the ones of Ref. [12] obtained by direct numerical solution of the Schrödinger equation (10) with boundary conditions (19). Many-channel variational results obtained in Refs. [26] and [27] are also presented for comparison. It is remarkable that numbers of bound states obtained in the frame of the adiabatic and B-O approximations are the same for each $J$. However, adiabatic binding energies are shifted below the corresponding $\mathrm{B}-\mathrm{O}$ ones. The influence of adiabatic corrections is minimal for the $J=0$ ground-state energy, $\varepsilon_{00}$, of $\left({ }^{4} \mathrm{He} \mu \mathrm{t}\right)^{++}$molecule, whereas it is maximal for the $J=2$ ground-state energy, $\varepsilon_{20}$, of $\left({ }^{3} \mathrm{He} \mu \mathrm{d}\right)^{++}$.

\footnotetext{
${ }^{\ddagger}$ Binding energy, $\varepsilon_{01}$, of the loosely bound state of $\left({ }^{4} \mathrm{He} \mu \mathrm{t}\right)^{++}$molecule is not presented in Ref. [12].
} 


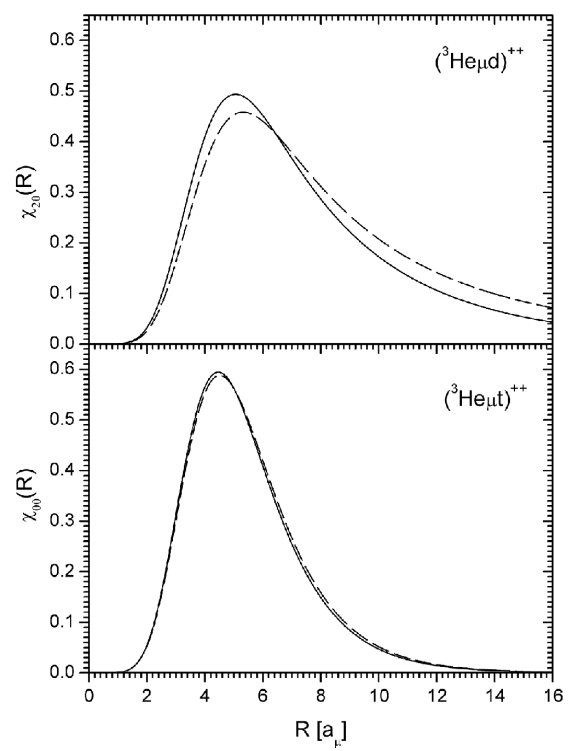

Fig. 13. Comparison of nuclear wave function $\chi_{20}(R)$ (top) for $\left({ }^{3} \mathrm{He} \mu \mathrm{d}\right)^{++}$molecule and $\chi_{00}(R)$ (bottom) for $\left({ }^{3} \mathrm{He} \mu \mathrm{t}\right)^{++}$molecule, calculated with inclusion of adiabatic corrections (solid) with their Born-Oppenheimer counterparts (dashed line).

\section{TABLE III}

Energy levels of $(\mathrm{He} \pi \mathrm{h})^{++}$molecules calculated in the one-level adiabatic (Ad.) and the Born-Oppenheimer (B-O) approximation obtained in the present paper.

\begin{tabular}{c|c|c|c|c|c|c|c}
\hline \hline$-\varepsilon_{J v}$ & & ${ }^{3} \mathrm{He} \pi \mathrm{p}$ & ${ }^{4} \mathrm{He} \pi \mathrm{p}$ & ${ }^{3} \mathrm{He} \pi \mathrm{d}$ & ${ }^{4} \mathrm{He} \pi \mathrm{d}$ & ${ }^{3} \mathrm{He} \pi \mathrm{t}$ & ${ }^{4} \mathrm{He} \pi \mathrm{t}$ \\
\hline$-\varepsilon_{00}$ & Ad. & 79.29 & 89.32 & 78.27 & 90.30 & 79.61 & 92.82 \\
& B-O & 38.22 & 41.44 & 62.87 & 68.53 & 75.37 & 82.51 \\
\hline$-\varepsilon_{10}$ & Ad. & 27.89 & 38.30 & 41.86 & 55.42 & 48.96 & 64.21 \\
& B-O & 2.74 & 6.19 & 30.10 & 37.73 & 45.74 & 55.46 \\
\hline$-\varepsilon_{20}$ & Ad. & & & & & & 14.59 \\
& B-O & & & & & & 9.27
\end{tabular}

Figures 13 present a comparison of the corresponding nuclear wave functions calculated with and without inclusion of adiabatic corrections.

It is interesting to examine also the influence of adiabatic corrections on bound-state energies of hydrogen-helium molecules, in which the muon is replaced by negative pion, $\pi^{-}$. The corresponding binding energies calculated with and without inclusion of adiabatic corrections are presented in Table III (according to our best knowledge, these results are presented for the first time). Due to less $M / m_{\mathrm{a}}$ mass ratios the influence of the corrections on energy levels is much more 


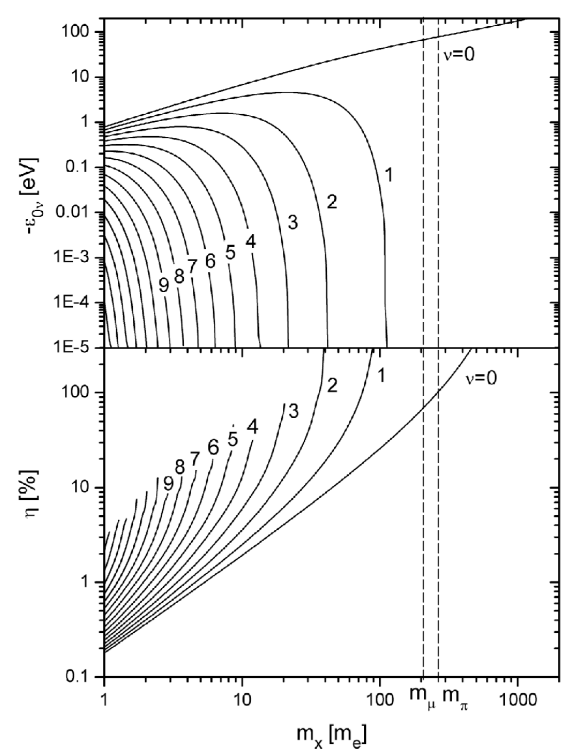

Fig. 14. Bound state energies (top) corresponding to $J=0$ of fictitious $\left({ }^{3} \text { Hexp }\right)^{++}$ molecule calculated in the one-level adiabatic approximation vs. the mass of a fictitious negative particle $\mathrm{x}$. Mass-dependence of the corresponding relative difference between adiabatic and $\mathrm{B}-\mathrm{O}$ energy levels, $\eta=\left(\varepsilon_{0 v}^{\mathrm{A}}-\varepsilon_{0 v}^{\mathrm{BO}}\right) / \varepsilon_{0 v}^{\mathrm{BO}}$, is also presented (bottom).

pronounced for pionic systems than for muonic ones. The same is true for nuclear wave functions, as expected.

Figure 14 presents energy levels (top) corresponding to $J=0$ of a fictitious molecule $\left({ }^{3} \mathrm{Hexp}\right)^{++}$calculated in the adiabatic one-level approximation vs. mass $m_{\mathrm{x}}$ of a fictitious negative particle $\mathrm{x}$. Mass $m_{\mathrm{x}}$ changes from electron mass to anti-proton mass. The corresponding relative difference between the adiabatic and $\mathrm{B}-\mathrm{O}$ energy levels (bottom), $\eta=\left(\varepsilon_{0 v}^{\mathrm{A}}-\varepsilon_{0 v}^{\mathrm{BO}}\right) / \varepsilon_{0 v}^{\mathrm{BO}}$, increases with increasing $m_{\mathrm{x}}$. For a given $m_{\mathrm{x}}, \eta$ sharply increases with increasing vibrational quantum number $v$. Points of intersections between vertical lines, corresponding to muon mass and pion mass, and lines of the diagram correspond to binding energies of the respective muonic and pionic molecules for $J=0$ presented in Tables II and III, and to the relative energy differences.

\section{Conclusions}

In this paper we present total and partial cross-sections for elastic $(\mathrm{h} \mu)_{1 s}+$ $\mathrm{He}^{++}$scattering calculated in the one-level adiabatic and Born-Oppenheimer approximations for the $2 p \sigma$ molecular state. Our adiabatic cross-sections significantly differ from the corresponding results of Ref. [30], obtained using the same method of calculation, and are close to the ones of Ref. [29] (the two-level calculation). At the same time, the existing discrepancy between results of Ref. [29] and [30] 


$$
\text { Elastic }(h \mu)_{1 s}+H e^{++} \text {Scattering } \ldots
$$

is resolved in favor of the first paper. We also calculated scattering lengths, and positions and widths of resonances appearing in elastic $(\mathrm{h} \mu)_{1 s}+\mathrm{He}^{++}$scattering for collision energy range $0 \leq \varepsilon \leq 50 \mathrm{eV}$. It was shown that adiabatic corrections to the Born-Oppenheimer scattering potential are responsible for proper positions of the Ramsauer-Townsend minima in slow $(\mathrm{p} \mu)_{1 s}+{ }^{3,4} \mathrm{He}^{++}$collisions. The influence of the corrections on energy levels of muonic, $(\mathrm{He} \mu \mathrm{h})^{++}$, and pionic, $(\mathrm{He} \pi \mathrm{h})^{++}$, molecules is also presented. As is seen from Tables II, III and Fig. 14, the influence of adiabatic corrections decreases with increasing mass ratio, $M / m_{\mathrm{a}}$, and decreasing coefficient $\kappa$. This observation corresponds with the form of potential $V_{\mathrm{A}}(R)$ expressed by Eqs. (11)-(14).

\section{Acknowledgments}

The State Committee for Scientific Research is greatly acknowledged for financial support of the work. One of us (N.P.) would like to thank Profs. K. Jelen and S. Taczanowski for the kind hospitality during his stay in Kraków at the Faculty of Physics and Applied Computer Science, UST.

\section{References}

[1] F.C. Frank, Nature 160, 525 (1947); A.D. Sakharov, Lebedev Physics Institute Report, 1948, unpublished; Muon Catal. Fusion 4, 235 (1989); L.W. Alvarez, H. Bradner, F.S. Crawford Jr., J.A. Crawford, P. Falk-Vairant, M.L. Good, J.D. Gow, A.H. Rosenfeld, F. Solmitz, M.L. Stevenson, H.K. Ticho, R.D. Tripp, Phys. Rev. 105, 1127 (1957); E.A. Vesman, Pis'ma Zh. Eksp. Teor. Fiz. 5, 113 (1967) [Sov. Phys. JETP Lett. 5, 91 (1967)]; W.H. Breunlich, P. Kammel, J.S. Cohen, M. Leon, Ann. Rev. Nucl. Part. Sci. 39, 311 (1989); C. Petitjean, Nucl. Phys. A 543, 79c (1992); J.S. Cohen, RIKEN Review 20, 8 (1999).

[2] L. Bracci, G. Fiorentini, Phys. Rep. 86, 169 (1982); A. Guła, Acta Phys. Pol. B 16, 589 (1985); P. Froelich, Adv. Phys. 41, 405 (1992).

[3] V.B. Belyaev, V.I. Korobov, S.A. Rakityansky, Few-Body Systems 17, 243 (1994).

[4] A. Bertin, A. Vitale, in: Fifty Years of Weak-Interaction Physics, Eds. A. Bertin, R.A. Ricci, A. Vitale, Italian Physical Society, Bologna 1984, p. 130; V.M. Bystritsky, V.F. Boreiko, M. Filipowicz, V.V. Gerasimov, O. Huot, P.E. Knowles, F. Mulhauser, V.N. Pavlov, L.A. Schaller, H. Schneuwly, V.G. Sandukovsky, V.A. Stolupin, V.P. Volnykh, J. Woźniak, Phys. Rev. A 69, 012712 (2004).

[5] J.S. Cohen, R.L. Martin, W.R. Wadt, Phys. Rev. A 24, 33 (1981); Phys. Rev. A 27, 1821 (1983); Phys. Rev. A 27, 167 (1983); J.D. Garcia, N.H. Kwong, J.S. Cohen, Phys. Rev. A 35, 4068 (1987); N.H. Kwong, J.D. Garcia, J.S. Cohen, J. Phys. B, At. Mol. Opt. Phys. 22, L633 (1989); G.Y. Korenman, Hyperfine Interact. 101/102, 81 (1996); G.A. Fesenko, G.Y. Korenman, Hyperfine Interact. 101/102, 91 (1996); T.S. Jensen, V.E. Markushin, Hyperfine Interact. 138, 71, $113(2001)$. 
[6] L.I. Menshikov, L.I. Ponomarev, Z. Phys. D 2, 1 (1986); A.V. Kravtsov, N.P. Popov, Z. Phys. D 6, 61 (1987); A.V. Kravtsov, A.I. Mikhailov, N.P. Popov, Sov. Phys. JETP 69, 246 (1989) [Zh. Eksp. Teor. Fiz. 96, 437 (1989)]; A. Gula, A.V. Kravtsov, A.I. Mikhailov, Z. Oziewicz, N. Popov, Muon Catal. Fusion 4, 217 (1989); A.V. Kravtsov, A.I. Mikhailov, Phys. Rev. A 49, 3566 (1994); A.V. Kravtsov, A.I. Mikhailov, Phys. Rev. A 58, 4426 (1998); E.C. Aschenauer, V.E. Markushin, Z. Phys. D 39, 165 (1997); W. Czapliński, A. Kravtsov, A. Mikhailov, N. Popov, Acta Phys. Pol. A 93, 617 (1998).

[7] S. Cohen, D.L. Judd, R.J. Riddell, Phys. Rev. 119, 397 (1960).

[8] A.V. Kravtsov, A.I. Mikhailov, N.P. Popov, Phys. Lett. A 116, 180 (1986).

[9] N.P. Popov, Muon Catal. Fusion 2, 207 (1988); A. Adamczak, V.S. Melezhik, L.I. Menshikov, Z. Phys. D 4, 153 (1986); A. Adamczak, V.S. Melezhik, Muon Catal. Fusion 2, 131 (1988); J.S. Cohen, Muon Catal. Fusion 5/6, 3 (1990/91); V. Bystritsky, W. Czaplinski, J. Woźniak, E. Guła, A. Kravtsov, A. Mikhailov, N. Popov, Phys. Rev. A 53, 4169 (1996).

[10] M. Leon, H.A. Bethe, Phys. Rev. 127, 636 (1962); A.P. Bukhvostov, N.P. Popov, Zh. Eksp. Teor. Fiz. 82, 23 (1982) [Sov. Phys. JETP 55, 13 (1982)]; L.I. Menshikov, Muon Catal. Fusion 2, 173 (1988); V. Bystritsky, W. Czaplinski, N. Popov, Eur. Phys. J. D 5, 185 (1999).

[11] L. Bracci, G. Fiorentini, Nuovo Cimento A 43, 9 (1978); W. Czaplinski, A. Gula, A. Kravtsov, A. Mikhailov, N. Popov, Phys. Rev. A 50, 525 (1994); L.I. Ponomarev, E.A. Solov'ev, Pis'ma Zh. Eksp. Teor. Fiz. 64, 129 (1996) [JETP Lett. 64, 135 (1996)]; A.V. Kravtsov, A.I. Mikhailov, Zh. Eksp. Teor. Fiz. 107, 1473 (1995) [Sov. Phys. JETP 80, 822 (1995)]; A.V. Kravtsov, A.I. Mikhailov, Phys. Rev. A 58, 4426 (1998); A.V. Kravtsov, A.I. Mikhailov, L.I. Ponomarev, E.A. Solovyov, Hyperfine Interact. 138, 103 (2002).

[12] Yu.A. Aristov, A.V. Kravtsov, N.P. Popov, G.E. Solyakin, N.F. Truskova, M.P. Faifman, Yad. Fiz. 33, 1066 (1981) [Sov. J. Nucl. Phys. 33, 564 (1981)].

[13] W. Czaplinski, A. Kravtsov, A. Mikhailov, N. Popov, Phys. Lett. A 219, 86 (1996); W. Czaplinski, A. Kravtsov, A. Mikhailov, N. Popov, Eur. Phys. J. D 3, 223 (1998).

[14] F.M. Pen'kov, Yad. Fiz. 60, 1003 (1997) [Phys. At. Nucl. 60, 897 (1997)]; L.N. Bogdanova, V.I. Korobov, L.I. Ponomarev, Hyperfine Interact. 118, 183 (1999).

[15] I.V. Komarov, L.I. Ponomarev, S.Yu. Slovianov, Spheroidal and Coulomb Spheroidal Functions, Nauka, Moscow 1976, p. 318.

[16] S.I. Vinitsky, L.I. Ponomarev, Fiz. Elem. Chastits At. Yadra 13, 1336 (1982) [Sov. J. Part. Nucl. 13, 557 (1982)].

[17] J.H. Macek, J. Phys. B 1, 831 (1968); M. Fabre de la Ripelle, M.I. Haftel, S.Y. Larsen, Phys. Rev. A 44, 7084 (1991); V.V. Gusev, V.I. Puzynin, V.V. Kostrykin, A.A. Kvitsinsky, S.P. Merkuriev, L.I. Ponomarev, Few-Body Systems 9, 137 (1990); M. Decker, W. Sandhas, V.B. Belyaev, Phys. Rev. A 53, 726 (1996).

[18] K.K. Sabelfeld, Monte Carlo Methods in Boundary Value Problems, Springer-Verlag, Berlin 1991, p. 283; D.M. Caperley, B.J. Alder, Phys. Rev. A 31, 1999 (1985); S.P. Merkuriev, S.A. Niemnugin, Few-Body Systems 14, 191 (1993). 
[19] L.D. Faddeev, Mathematical Aspects of the Three-Body Problem in Quantum Scattering Theory, Steklov Math. Inst., Leningrad 1963 [Engl. transl. D. Davey and Co., New York 1965]; A.A. Kvitsinsky, Chi-Yu Hu, J.S. Cohen, Phys. Rev. A 53, 255 (1996); Chi-Yu Hu, A.A. Kvitsinsky, Phys. Rev. A 46, 7301 (1992); Chi-Yu Hu, A.A. Kvitsinsky, S.P. Merkuriev, Phys. Rev. A 45, 2723 (1992).

[20] Y. Kino, M. Kamimura, Hyperfine Interact. 82, 195 (1993).

[21] O.I. Kartavtsev, V.I. Kochin, E.A. Kolganova, Hyperfine Interact. 118, 235 (1999).

[22] V.I. Korobov, Hyperfine Interact. 101/102, 329 (1996).

[23] E. Hiyama, Y. Kino, M. Kamimura, Prog. Part. Nucl. Phys. 51, 223 (2003).

[24] V.M. Bystritsky, V.P. Dzhelepov, V.I. Petrukin, A.I. Rudenko, V.M. Suvorov, V.V. Filtchenkov, N.N. Hovansky, B.A. Homenko, Zh. Eksp. Teor. Fiz. 84, 1257 (1983) [Sov. Phys. JETP 57, 728 (1983)]; D.V. Balin, A.A. Vorobyov, An.A. Vorobyov, Yu.K. Zalite, A.A. Markov, V.I. Medvedev, E.M. Maev, G.G. Semenchuk, Yu.V. Smirenin, Pis'ma Zh. Eksp. Teor. Fiz. 42, 236 (1985) [JETP Lett. 42, 293 (1985)]; T. Matsuzaki, K. Ishida, K. Nagamine, Muon Catal. Fusion 2, 217 (1988); V.M. Bystritsky, A.V. Kravtsov, J. Rak, Kerntechnik 58, 185 (1993); S. Tresh, R. Jacot-Guillarmod, F. Mulhauser, C. Piller, L.A. Schaller, L. Schellenberg, H. Schneuwly, Y.-A. Thalmann, A. Werthmüller, Phys. Rev. A 57, 2496 (1998); B. Gartner, P. Ackerbauer, W.H. Breunlich, M. Cargnelli, P. Kammel, R. King, B. Lauss, J. Marton, W. Prymas, J. Zmeskal, C. Petitjean, M. Augsburger, D. Chatellard, J.-P. Egger, T. von Egidy, F.J. Hartmann, A. Kosak, M. Mühlbauer, F. Mulhauser, L.A. Schaller, L. Schellenberg, H. Schneuwly, Y.-A. Thalmann, S. Tresch, A. Werthmüller, Phys. Rev. A 62, 012501 (2000); M. Augsburger, P. Ackerbauer, W.H. Breunlich, M. Cargnelli, D. Chatellard, J.-P. Egger, B. Gartner, F.J. Hartmann, O. Huot, R. Jacot-Guillarmod, P. Kammel, R. King, P. Knowles, A. Kosak, B. Lauss, J. Marton, M. Mühlbauer, F. Mulhauser, C. Petitjean, W. Prymas, L.A. Schaller, L. Schellenberg, H. Schneuwly, S. Tresch, T. von Egidy, Phys. Rev. A 68, 022712 (2003).

[25] Chi-Yu Hu, A.K. Bhatia, Phys. Rev. A 45, 5769 (1990); Chi-Yu Hu, A.K. Bhatia, Muon Catal. Fusion 5/6, 439 (1991).

[26] J. Wallenius, P. Froelich, Hyperfine Interact. 118, 223 (1999).

[27] V.B. Belyaev, O.I. Kartavtsev, V.I. Kochin, E.A. Kolganova, Z. Phys. D 41, 239 (1997); V.B. Belyaev, O.I. Kartavtsev, V.I. Kochin, E.A. Kolganova, Phys. Rev. A 52, 1765 (1995).

[28] S.S. Gerstein, V.V. Gusev, Hyperfine Interact. 82, 185 (1993).

[29] A.V. Matveenko, L.I. Ponomarev, Zh. Exp. Teor. Fiz. 63, 48 (1972) [Sov. Phys. JETP 36, 24 (1973)].

[30] A.V. Kravtsov, A.I. Mikhailov, N.P. Popov, J. Phys. B, At. Mol. Phys. 19, 1323 (1986).

[31] A. Kravtsov, A. Mikhailov, N. Popov, Phys. Lett. A 223, 129 (1996).

[32] S.S. Gerstein, Zh. Eksp. Teor. Fiz. 43, 706 (1962) [Sov. Phys. JETP 16, 501 (1963)]. 
[33] A.R. Edmonds, Angular Momentum in Quantum Mechanics, Princeton University Press, Princeton 1957, p. 146.

[34] L.I. Schiff, Quantum Mechanics, 3rd ed., McGraw-Hill, New York 1968, p. 544.

[35] M. Abramowitz, I.A. Stegun, Handbook of Mathematical Functions with Formulas, Graphs, and Mathematical Tables, Wiley, New York 1972, p. 1046.

[36] F. Calogero, Variable Phase Approach to Potential Scattering, Academic Press, New York 1967, p. 244; V.V. Babikov, Method Fazovyh Funkcij v Kvantovoj Mehanike, 2nd ed., Nauka, Moskva 1988, p. 288 (in Russian).

[37] R.G. Newton, Scattering Theory of Waves and Particles, 2nd ed., Springer-Verlag, New York 1982, p. 743.

[38] H.C. Ohanian, C.G. Ginsburg, Am. J. Phys. 42, 310 (1974).

[39] L.D. Landau, Quantum Mechanics: Non-relativistic Theory, Pergamon Press, London 1958, p. 515.

[40] P.G. Burke, Potential Scattering in Atomic Physics, Plenum Press, New York 1977, p. 138.

[41] A. Bohm, Quantum Mechanics - Foundations and Applications, 3rd ed., Springer-Verlag, New York 1993, p. 479. 